\title{
Ancient Vedic Multiplication Based Optimized High Speed Arithmetic Logic
}

\author{
Mrs. Leena Rathi \\ Department of Management Studies, Khamgoan India \\ leenarathi@gmail.com
}

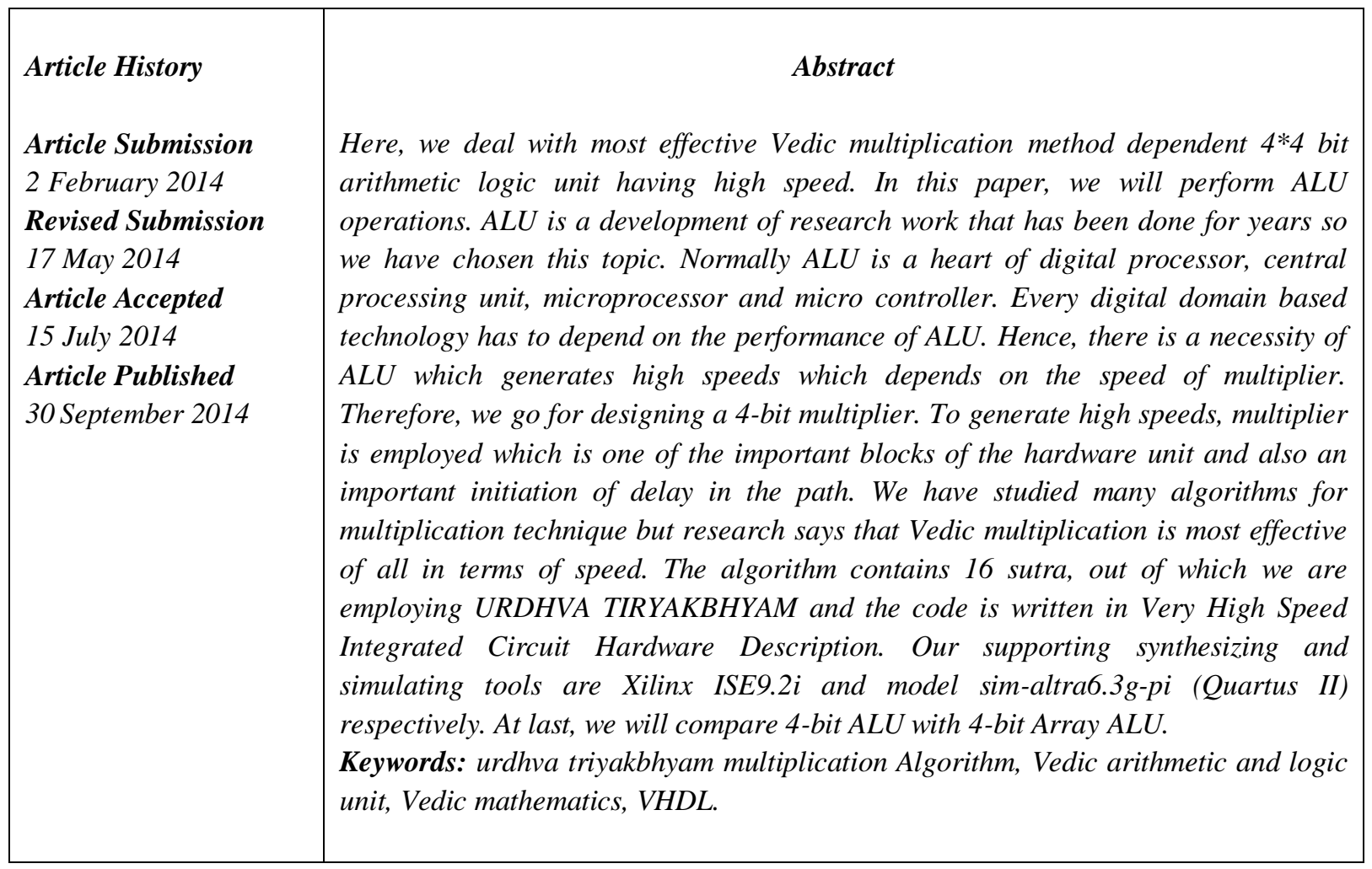

\section{Introduction}

Already, we have studied that ALU is a mathematical unit and executes arithmetic \& logical operations. Hence, ALU is the heart of $\mu$ processor, $\mu$ controller and DSP. Here, not only executes arithmetic functions but also carryout logical functions. It can execute three arithmetic and five logical functions. In recent days, high speed processors are becoming more and more. ALU speed is the main important feature of digital domain based system. The ALU is used to perform partially or whole in all the technologies and the ALU speed mostly depend upon multiplier speed. Therefore, we go for developing a 4-bit Vedic multiplier having more speeds. This multiplier is very fast and less hardware components are required and shown in fig 1.

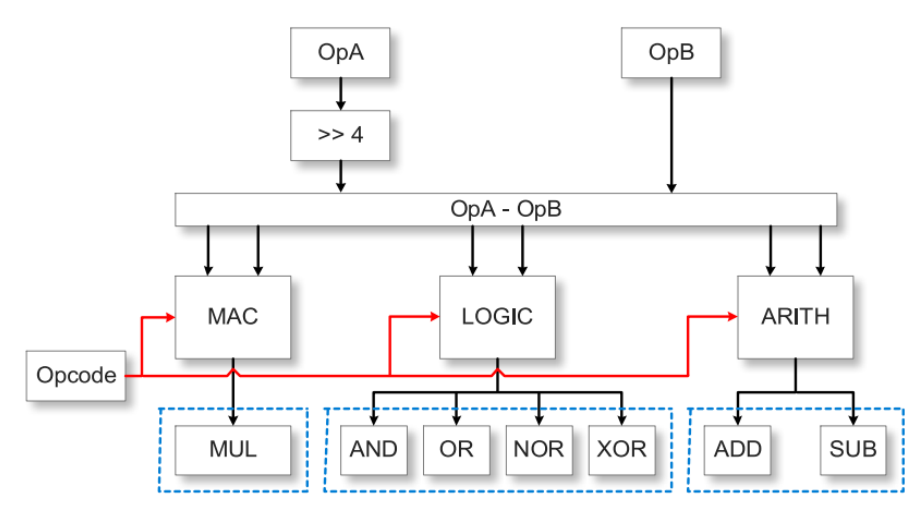

Fig 1: Basic Blocks in ALU Architecture 


\section{Related Work}

The ancient Vedic mathematic is a gift given to Indian sages. Hinder cleric wrote Vedic mathematics. It contains mental calculation techniques related to Vedas. This is described in Vedic work. The Vedic mathematics uses a large number of calculations are done within a short time. The Vedic mathematics is based on the normal human mind working principle. The Vedic mathematic perform high speed calculations used in the engineering field for performing effective algorithm. Multiplier dependent 4-bit Vedic ALU is discussed here. We are going to employ an algorithm called as urdhva tiryakbhyam procedure. It is employed in decimal system to product two numbers. Here, proposed method develops the same in binary system. Hence, it is generalized for all multiplication purposes which is adopted for digital hardware units. Multiplier adopts the sutra called as partial protect where the sums are evaluated in parallel. Advantages of the above discussed method are increment in bits, area and decrement in speed.

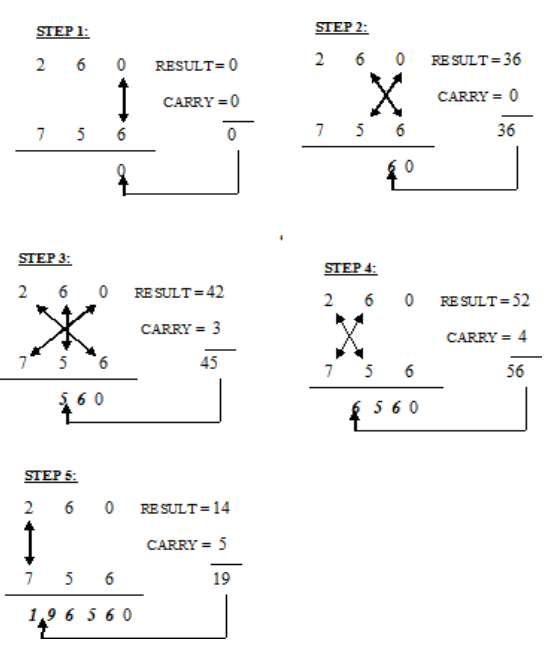

Fig 2: schematic representation of multiplication of two decimal numbers

We will now adopt this algorithm for binary number system. The binary multiplication technique multiplies two bits which is just an AND operation. Let us consider, two 4-bit binary numbers such as $A=1011$ and $B=1101$.It produces 8-bit binary number by using vertically and crosswise method. The example of 4-bit binary multiplication technique in urdhva thriyakbhyam method is given below in figure 3 .
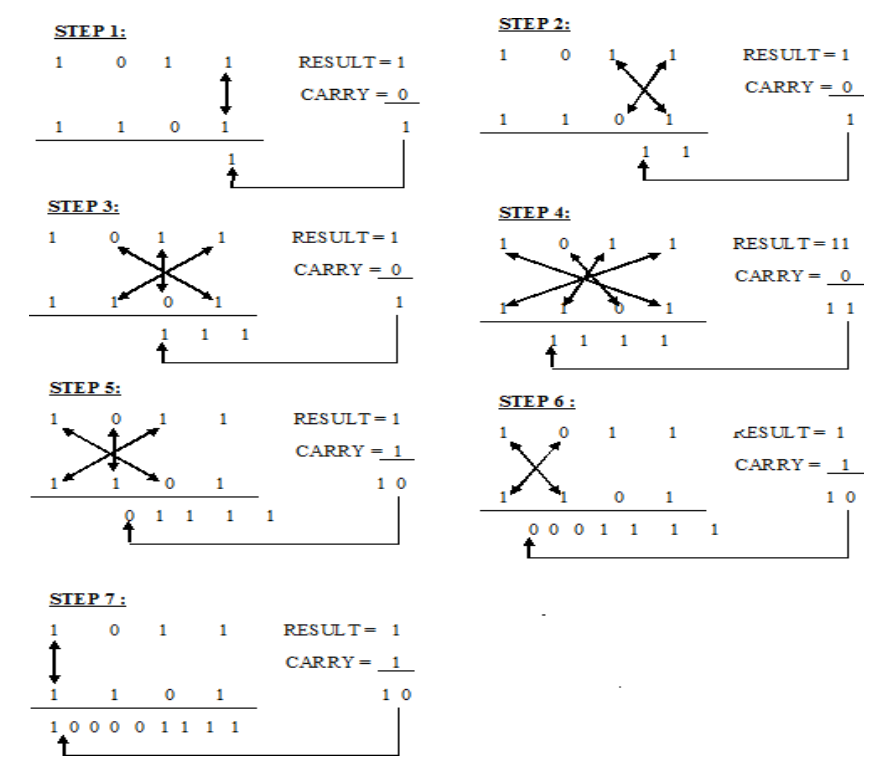

Fig 3: Schematic representation of multiplication of two 4-bit binary numbers 


\section{Proposed Data path Designs}

Here, we discuss in detail about two $2 \times 2$ bit, $4 \times 4$ bit are observed. We also employ urdhva tiryakbhyam in multiplying two numbers. The benefit of this method is the result of partial products and done addition in parallel. In this section explained multiplied two 2- bit numbers in urdhva tiryakbhyam method. This is already explain above section and now let us consider two 2-bit number $\mathrm{X}=\mathrm{x} 1 \mathrm{x} 2$ and $\mathrm{Y}=\mathrm{y} 1 \mathrm{y} 2$. The implementation equation of $2 \times 2$ Vedic multiplier are

$$
\begin{aligned}
& \text { S0 }=x 0 y 0=A \\
& \text { C1 S1 }=x 0 y 1+y 0 x 1=B \\
& \text { C2 S2 }=\text { C1+x1y1=C }
\end{aligned}
$$

The final result is DCBA. The D is specified by carry bit. Here the implemented 2-half adder modules as given below in figure 4 . The two half adders used here generate their own path delays and shown in fig 4. .

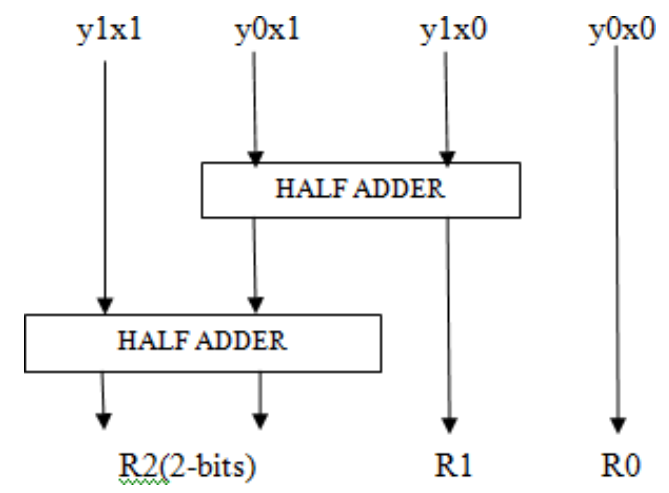

Fig 4: Schematic representation of $2 X 2$ multiplier module

A 4-bit Vedic multiplier is designed by employing AND gate, adder in this paper. It contains full adders eight in number, half adder three in number, AND gates sixteen in number. The main purpose is to decrement speed. Below figure shows the architecture. We take into consideration $4 \times 4$ multiplication $\mathrm{X}=\mathrm{x} 3 \mathrm{x} 2 \mathrm{x} 1 \mathrm{x} 0$ and $\mathrm{Y}=\mathrm{y} 3 \mathrm{y} 2 \mathrm{y} 1 \mathrm{y} 0$. An eight bit plus carry bit is the output generated. The result is given by $\mathrm{P}=\mathrm{p} 7 \mathrm{p} 6 \mathrm{p} 5 \mathrm{p} 4 \mathrm{p} 3 \mathrm{p} 2 \mathrm{p} 1 \mathrm{p} 0$ and is shown in fig 5 .

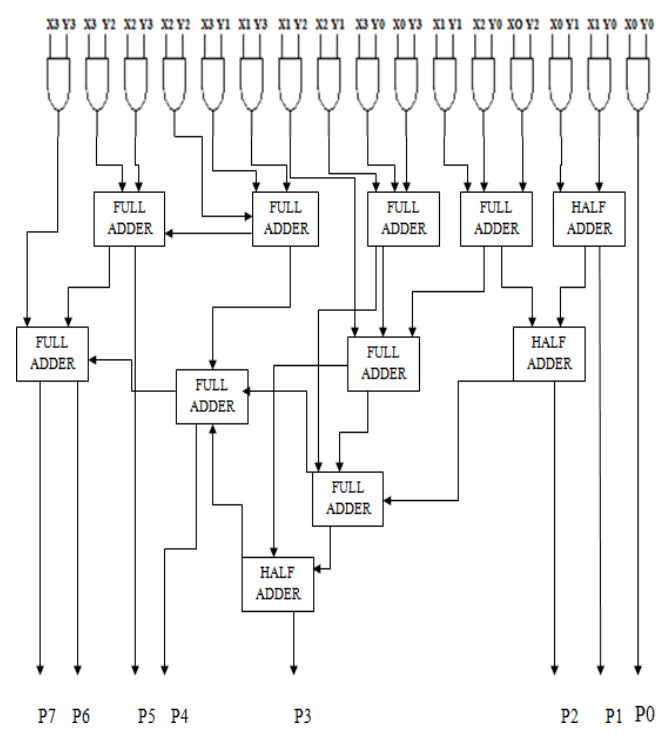

Fig 5: block diagram of $4 \times 4$ multiplier module 
Figure 6 shows a Vedic 4-bit architecture which is proposed here. Dependent on selection lines inputs A and B executes ALU functions. We here employ 8:1 and two 2:1 multiplexer. Input B, 2's complement are received by 2:1 multiplexer. If all the selection lines are zero, A is added with B directly, or else, A and input of 2's complement together added to produce output subtraction. All the arithmetic functions at the output are received by 8:1, employing selection lines logical functions are produced at the output. Logical functions output may be excess 4-bit that is of two state. Logical operations attained will observed in output of LSB. Arithmetic functions output may be also excess 4-bit and is observed in MSB.

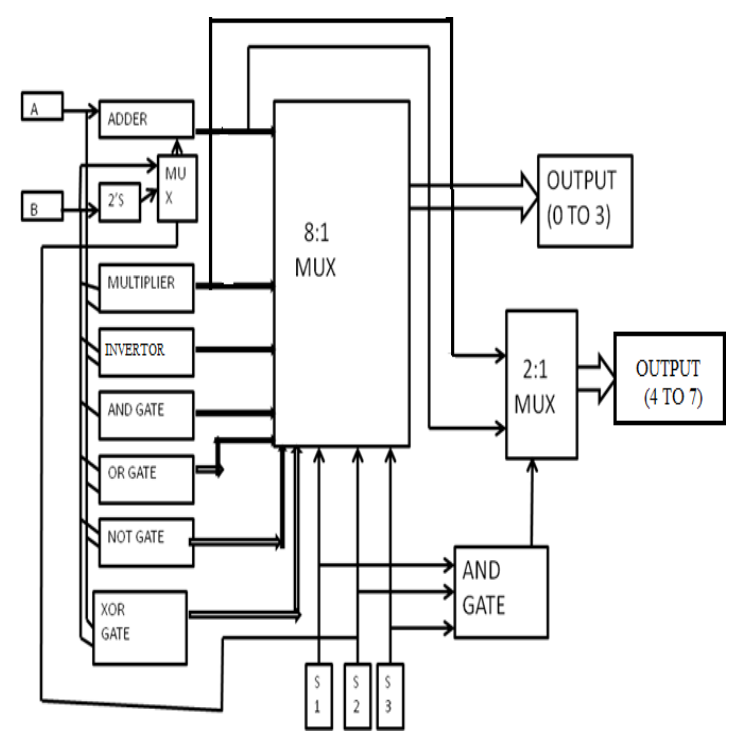

Fig 6: Schematic representation of the 4-bit vedic ALU architecture

\section{Simulation Results}

In this paper, $4 \times 4$ bit Vedic multiplier and ALU are coded by employing VHDL. Xilinx ISE12.1i project navigator to simulate VHDL code. The four bits given below are tested by employing 4-bit multiplier simulator. Inputs of 4-bit multiplier are $\mathrm{A}=1111, \mathrm{~B}=1111$ and benefited output is $\mathrm{C}=11100001$ and is shown in figure 7 and figure 8 respectively.

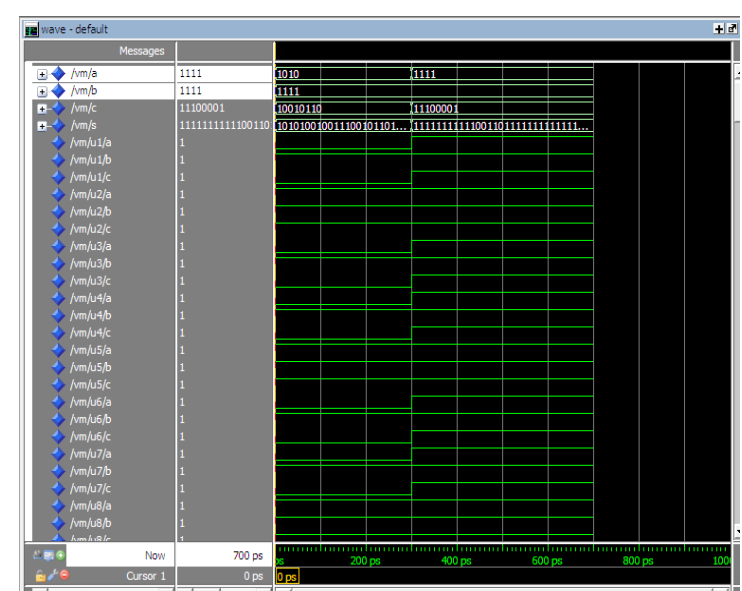

Fig 7: Simulation of 4-bit vedic multiplier 


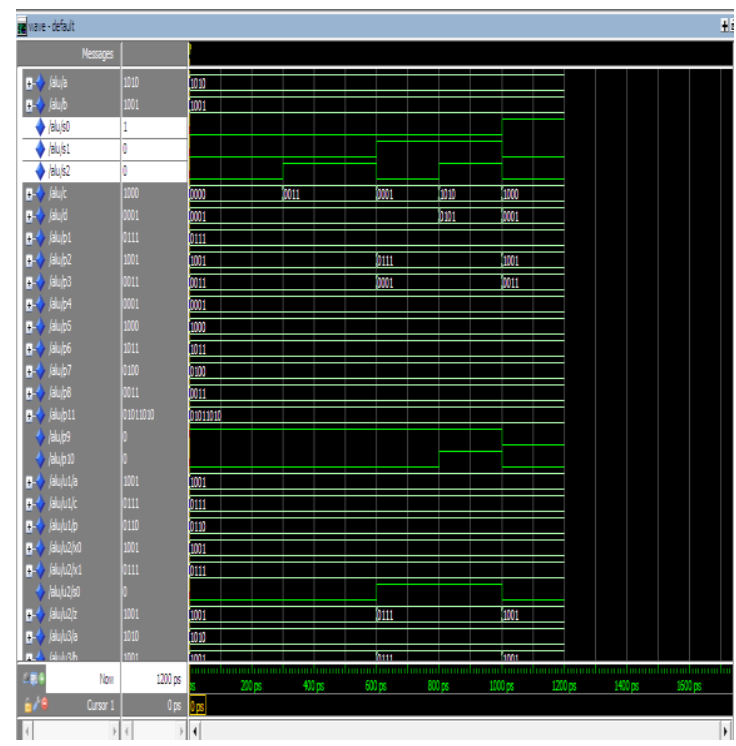

Fig 8: Simulation of 4-bit ALU

The inputs of selection lines are considered to be zero. After that addition function is chosen. Considered inputs are $A=0011, B=1001$ and the output generated is $C=0011, D=0001$. The inputs of selection lines are considered to be zero except $\mathrm{S} 3=1$. After that subtraction function is chosen. Considered inputs are $\mathrm{A}=1010$, $\mathrm{B}=1001$ and the output generated is $\mathrm{C}=0001, \mathrm{D}=0001$. From the table below, we verify that the Vedic multiplier has the better speed in comparison with multipliers. We have also designed all of this multiplier. Then this delay time is compared to our Vedic multiplier, which produces better time delays. The results are tabulated in table 1 .

TABLE 1: Comparison of Delay in Multiplier

\begin{tabular}{|c|c|c|c|c|}
\hline Multipliers & $\begin{array}{c}\text { Number of bit } \\
\text { slices }\end{array}$ & Number of IOs & $\begin{array}{c}\text { Number of } \\
\text { LUTs }\end{array}$ & Delay (ns) \\
\hline $\begin{array}{c}\text { Array } \\
\text { Multiplier }\end{array}$ & 40 & 20 & 45 & 20.11 \\
\hline $\begin{array}{c}\text { Vedic } \\
\text { Multiplier }\end{array}$ & 18 & 16 & 31 & 17.47 \\
\hline
\end{tabular}

Below table 2 shows the differentiate between 4-bit Vedic ALU and array ALU in terms of their speeds. Simulation results show that array ALU less path delay in comparison with Vedic ALU. This 4-bit array ALU and it delay time will compared.

TABLE 2: Comparison of Delay in Multiplier

\begin{tabular}{|c|c|c|c|c|}
\hline Multipliers & $\begin{array}{c}\text { Number of bit } \\
\text { slices }\end{array}$ & Number of IOs & $\begin{array}{c}\text { Number of } \\
\text { LUTs }\end{array}$ & Delay (ns) \\
\hline $\begin{array}{c}\text { Array } \\
\text { Multiplier }\end{array}$ & 29 & 19 & 51 & 17.58 \\
\hline $\begin{array}{c}\text { Vedic } \\
\text { Multiplier }\end{array}$ & 49 & 23 & 65 & 20.22 \\
\hline
\end{tabular}




\section{Conclusion}

Multiplier design discussed above is an effective one dependent on urdhva tiryakbhyam sutra. In simulation process, this technique decreases the path delay. So, it has the capability of simulating the calculations with higher speeds in comparison with various methods adopted. A $4 \times 4$ multiplier produces a speed of $15.27 \mathrm{~ns}$ in this paper. Speed of 4-bit ALU is 17.583 that far less in comparison with Array ALU path delay. So, it is much better than array ALU. Simulation results show 23\% improvement in delay parameter and can be implemented in higher architectures.

\section{References}

[1] G.Ganesh Kumar V.Charishma,"Dsign of high speed Vedic multiplier using Vedic mathematic techniques", International journal of scientific and Research publication, volum2/ISSUE3/march 2012/ISSN 22503153.

[2] Krishnaveni D and Umarani T.G,"VLSI Implementation of Vedic multiplier with reduces delay", International journal of advanced technology and engineering research (IJATER), ISSN NO: 22503536/volume 2/ISSUE4/July 2012.

[3] S Q Dani," Ancient Indian mathematics -A conspectus”, general article, resonance, March 2012, sprinyer link.

[4] Anvesh Kumar, Ashish Raman,” Low power ALU design by Ancient mathematics “, 978-1- 142445586-7110, 2010 IEEE.

[5] Syed Azman bin Syed Ismail, Pumadai a/p Siva subramaniyam", Multiplication with the Vedic method", precede social and behavioral and behavioral sciences 8(2010) 129-133.sci Erse science direct, ELSEVIER.

[6] Harpreet singh Dhillon and Abhijitmitra," Reduced-Bit Multiplication Algorithm For Digital Arithmetic", International journal of computational and Mathematical science 2:2,2008.

[7] Amartya Kumar Dutla," Mathematics in ancient India", series article, April 2002 Springer link.

[8] Y. Bansal, C. Madhu and P. Kaur, "High speed vedic multiplier designs-A review," 2014 Recent Advances in Engineering and Computational Sciences (RAECS), Chandigarh, 2014, pp. 1-6, doi: 10.1109/RAECS.2014.6799502.

[9] R. Gupta, R. Dhar, K. L. Baishnab and J. Mehedi, "Design of high performance 16 bit multiplier using vedic multiplication algorithm with McCMOS technique," 2014 International Conference on Green Computing Communication and Electrical Engineering (ICGCCEE), Coimbatore, 2014, pp. 1-6, doi: 10.1109/ICGCCEE.2014.6922296.

[10] A. Bisoyi, M. Baral and M. K. Senapati, "Comparison of a 32-bit Vedic multiplier with a conventional binary multiplier," 2014 IEEE International Conference on Advanced Communications, Control and Computing Technologies, Ramanathapuram, 2014, pp. 1757-1760, doi: 10.1109/ICACCCT.2014.7019410.

[11] B. S. Premananda, S. S. Pai, B. Shashank and S. S. Bhat, "Design of area and power efficient complex number multiplier," Fifth International Conference on Computing, Communications and Networking Technologies (ICCCNT), Hefei, 2014, pp. 1-5, doi: 10.1109/ICCCNT.2014.6963017. 